\title{
Research of Human Resource Risk Warning Model of Dynamic Grey BP Neural Network based on Grey Relational Analysis Method
}

\author{
Ziyang Tang $^{1}$, Zhiqiang Wang ${ }^{1}$, Xuefang $\mathrm{Wei}^{1}$ \\ ${ }^{1}$ Wuhan University of Technology, HuBei, Wuhan, 430070 \\ hunter2011@foxmail.com
}

\begin{abstract}
Keywords: Risk Early Warning System of Human Resources; Gray Correlation Analysis; Gray BP Neural Network
\end{abstract}

\begin{abstract}
Against the backdrop of economic globalization and knowledge economy era, the role of human resources in the core competitiveness of enterprises has been increasingly prominent. However, due to the complex changing external environment and employee psychological, physiological complexity and diversity, the corporate human resource management activities always face the potential internal and external risks. Therefore, to correctly identify the risks and causes of human resources on the basis of risk assessment results, improve the accuracy and objectivity, thus proposing targeted administrative measures, it has important practical significance and has become a hot academic research subject. This paper summarizes the research results on the basis of domestic and foreign scholars, introduces into the dynamic gray BP neural network model based on gray relational analysis to develop a new method for warning of corporate human resources and improves human resource management.
\end{abstract}

\section{Introduction}

In recent years, knowledge and intellectual capital has become an important factor in the development of enterprises, human resources is increasingly becoming the core competitiveness impact of strategic resources. However, due to the possibility of changing business complex of internal and external environment influence, coupled with people's own psychology deviate organizations, individual behavior complexity and physiological differences caused by the objective of [1], making the company's human resource management Events always face the potential internal and external risks. Therefore, the risk of corporate human resources is warning, so that timely adoption and effective approach to risk management and control is particularly critical. Based on this, in order to improve the accuracy of early warning of human resources and scientific methods, we learn from the results of previous studies, based on the proposed dynamic gray BP neural network of human resources risk early warning model based on gray relational analysis.

\section{Human Resources Risk Warning Indicator System}

In this paper, with reference to the results of empirical research of scholars on the basis of theoretical analysis, expert design, questionnaire, most choose to use running, and constantly improve the logical route to design the index system. And according to the sensitivity of the principle of comprehensiveness, testability principle, comparability, qualitative and quantitative principles to develop six categories, a total risk incentives indicator system 27 specific indicators.

\section{Grey BP neural network of early warning model based on gray correlation analysis}

Model Procedure Details. Grey BP neural network model of gray correlation analysis of early warning based on the basic idea of this set up (Figure 2) as follows:

Processing key indicators: It is the main application of gray correlation analysis to determine the key indicator.

Human resource risk indicator data collection, now corporate life cycle is divided into entrepreneurial, growth, maturity and decline, at every stage of about three years [2], if the 
estimated time required for more than three years to re-gray correlation analysis, identification of key indicators, if within three years, the key indicator data directly as input layer of the neural network;

Construction of a tandem-type gray BP neural network: Build BP neural network based on historical data of actual, planned use of predictive indicators as input network to obtain the results as an early warning score.

Considering the convergence speed and precision standard deviation, to determine the optimal BP network topology [3];

The key indicator data as the input of neural network, expert ratings as output, while determining the number of hidden layer nodes, learning for each sample;

The use of actual historical data for network training, according to the learning accuracy and iteration number, adjust the weights between the layers, optimize the network;

The use of gray prediction model, to generate dynamic human resources risk indicators sequence forecast period;

The value of the input gray prediction BP neural network trained to calculate output, and warning.

Grey Theory Screening of Key Indicators. A key factor in human resource risk early warning system are subject to different types of businesses, affect different stages of the life cycle, the objective for effective real-time screening out the key indicators to determine gray correlation analysis of indicator data processing.

Grey correlation analysis steps are as follows:

A raw data nondimensionalization

In order to be evaluated as far as possible reflect the differences between the objects of, and thus take extreme value approach to the raw data were normalized operations. Formula Extreme approach to:

b calculate correlation coefficient:

$$
x_{i}=\frac{x_{i}-m_{j}}{M_{j}-m_{j}}
$$

$$
r\left(x_{0}, x_{i}\right)=\frac{\min _{i} \min _{k}\left|x_{0}(k)-x_{i}(k)\right|+\rho \max _{i} \max _{k}\left|x_{0}(k)-x_{i}(k)\right|}{\left|x_{0}(k)-x_{i}(k)\right|+\rho \max _{i} \max _{k}\left|x_{0}(k)-x_{i}(k)\right|}
$$

$c$ calculation of various factors associated with the main behavior is generally associated degree:

$$
r\left(x_{0}, x_{i}\right)=\frac{1}{n} \sum_{k=1}^{n 1} \xi(k)(k=1,2,3, \cdots, 9)
$$

BP neural network and gray prediction. BP neural network is a multi-layered network of "reverse push" learning algorithm includes two counter-propagating signals forward propagation and error process [4]. Firstly, forward propagation, the sample input of the input layer, hidden layer after effect through path connection weights and activation functions transferred to the output layer [5]. If the actual output with the expected output of the output layer does not match, then transferred back propagation, the error signal of neuronal connections along the original path back and adjust the connection weights of each neuron [6]. Forward and reverse the spread of the layers spread weight adjustment process is repeated until the network output error is reduced to an acceptable level, or to learn the number of pre-set date. Specific design steps and principles are as follows:

Input layer and output layer design: The number of BP neural input nodes moderation. Too much can cause the network structure is too large, inevitably introduce more noise information; too little can not guarantee the necessary information networks.

Hidden layers and nodes of choice: As Robert Hecht-Nielsen proved that any continuous function within a close range in 1989 BP network can be used to approximate a hidden layer, that is a three-layer BP network can continuous mapping is done arbitrarily complex dimensions, so the use of a single hidden layer modeling. And the number of hidden layer nodes, too little can weaken fault-tolerant network, too much will make the network training complex, extended learning time. 
For this reason, in the empirical formula $n_{1}=\sqrt{m+n}+a$ (for the input neuron number, the output neuron number, is a constant 1-10) is to determine the scope of the hidden layer, based on the number of units, considering the actual output and expected output vector network the mean square size and training steps to determine how much hidden nodes [7].

Grey prediction model for data missing information to predict the next gray causal conditions Incomplete [5]. According to a discrete number of random numbers can be generated through this process becomes significantly weakened compared with the randomness of a regular series of theory formation, the model has a strong feasibility. That model is based on the number of generated columns regularly been on the description of the process of change to do a long time, and to determine the coefficient differential equations, the eventual establishment of a differential equation model [8].

Collate and analyze models after the prediction step follows [9]:

A. once the original sequence to generate a cumulative 1 -AGO sequence:

$$
x^{(1)}(k)=\left\{x^{(1)}(1), x^{(1)}(2), \ldots x^{(1)}(n)\right\}
$$

B. univariate first order differential equations were fitted to establish albino equation:

.C. Time response type albino $\frac{d x^{(1)}}{d t}+a x^{(1)}=b$ equation

$$
\text { is } \hat{x}(t)=\left[x^{(0)}(1)-\frac{b}{a}\right] e^{-a(t-1)}+\frac{b}{a}
$$

D. reduction value

$$
\hat{x}^{(0)}(k+1)=\hat{x}^{(1)}(k+1)-\hat{x}^{(1)}(k)
$$

\section{Examples of Verification and Application of Early Warning Model}

W The company was founded in July 2009 and listed on the Shenzhen SME board in 2012, it is a professional networking technologies from research things, software development of high-tech companies. The company in May 2012 into the growth stage, as of August 2012 the company employs 1059 people. In this paper, a quarter of the monitoring period, collected the W company since August 2012 to 2015, human resource management data of a total of 12 cycles of August (Since the original data sample capacity, this article is not listed separately). In order to make the statistics of each index the same period, the daily, monthly monitoring of indicators, we used the average of the corresponding monitoring results as an indicator of the quarter.

In order to reflect a more intuitive enterprise risk status of human resources for the corresponding period, according to the experts to the degree of risk scores to be divided, divide the standard shown in Table 1:

Table 1 Risk division standard

\begin{tabular}{|c|c|}
\hline Score zone & Risk status \\
\hline $0-0.1$ & Very High \\
\hline $0.1-0.2$ & High \\
\hline $0.2-0.4$ & A little \\
\hline $0.4-0.6$ & Basic safe \\
\hline $0.6-0.8$ & safe \\
\hline $0.8-1.0$ & Much safe \\
\hline
\end{tabular}

This paper further use of gray relational analysis to select the key index, which calculate the results of the evaluation of early warning indicators gray correlation coefficient, select the order of relational degree before 20 indicators as a key index set.

Since then, we have selected the top ten cycle data normalized as input BP network, Experts rate as output, import MATLAB neural network toolbox, create and train a network [10].

Network structure and parameters finalized as follows:

A. Training function (Training Function): TRAINLM (Levenberg-Marquardt algorithm) 
B. learning function (Adaption Learning Function): TRAINGDM (gradient descent momentum BP algorithm function)

C. Performance function (Performance Function): MSE (mean square error)

D. Transfer function (Transfer Function): TANSIG (hyperbolic tangent transfer function S-type)

E. Neural Network Layers: 2

F. Hidden layer neuron number: 9

At the same time, drawn by the MATLAB Figure 1 shows when the network training has the sixth step, the network achieve optimal performance..

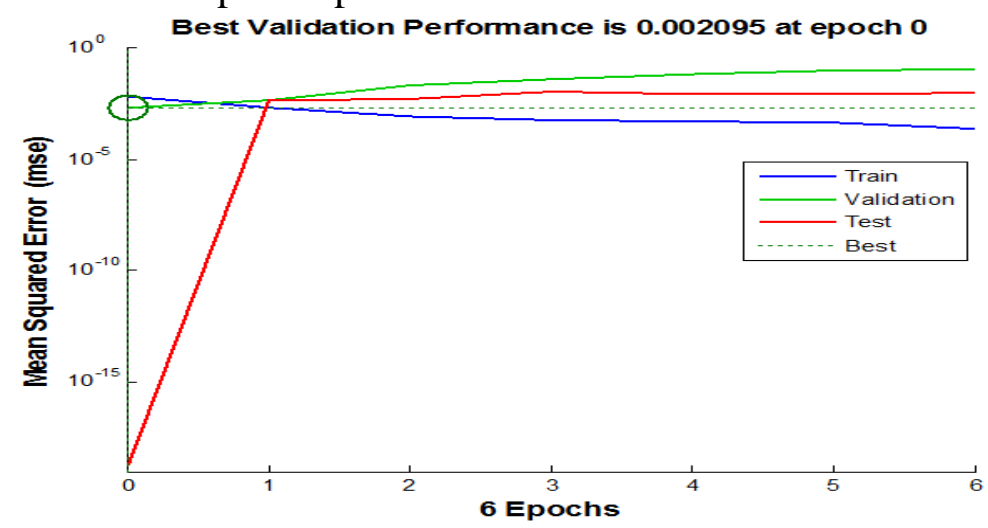

Fig. 1 Network error curve

After that, we use gray prediction method, as the experimental data for two cycles after the key index values and experts predict scores, as input and desired output value BP neural network. Meanwhile, in order to further strengthen the dynamic real-time early warning model, rolling forecasting and prediction data after two periods. Then, before the predicted results have been trained gray prediction method of input good BP neural network, to get the final results of human resource risk warning as shown in Table 2.

Table 2 Results Warning BP neural network output

\begin{tabular}{|l|l|l|l|}
\hline Network output & Score & Real Gap & Warning Result \\
\hline 0.62148 & 0.61 & $1.148 \mathrm{E}-02$ & safe \\
\hline 0.82991 & 0.82 & $9.91 \mathrm{E}-03$ & Much safe \\
\hline
\end{tabular}

\section{Conclusion}

Observe warning results above, it was found warning error prediction model output and the actual expert scores between small, early warning of a relatively high accuracy and precision. It explains the dynamic gray BP neural network model of everyday human enterprise risk warning applicability and effectiveness to some extent.

Through analysis, human resources risk warning indicator warning sign that there is an abstract model, alarm indicator, nonlinear function mapping relationship between the police of the approximation procedure [10]; and on the risk index, its data has poor regularity, difficult to quantify, fuzzy and strong [11] and other characteristics, and because of the various risk factors, it can lead to a crisis, and therefore the risk of human resources with a global early-warning feature [10]. The above uses gray BP neural network has good nonlinear function approximation and weaken the advantages of fluctuations in the data, randomness and prediction can better deal with the gray system features a strong global system, so apply at different life empirical research on human resource risk warning cycle businesses.

\section{References}

[1] N.D. Yang, Y.L. Zhang. Corporate Human Resource Risks and Countermeasures. Northwest University of Technology (Social Science), 1999,19 (2): 13-16.

[2] Y.H. Zhang. Enterprise Life Cycle Stages of Human Resource Management Research and Solutions to A Crisis Situation [D]. Southwest University of Finance and Economics. 2007. 
[3] Z.S. Liang, G.L. Zhang. Gray Correlation Calculation Method Comparison and Problem Analysis. Systems Engineering, 1996,143: 45-49.

[4] D.Q. Zhu, H. Shi. Neural Network Theory and Application. Science Press, 2006.

[5] R.H. Jiang, Q. Cao. Prediction Human Resource Needs of Enterprises based on Gray BP Neural Network [J]. Chinese Human Resources Development, 2011 (11): 42-46.

[6] Y.J. Guo. Evaluation Theory and Methods. Science and Technology Press, 2002.

[7] Y. Huang, H. Xiao. Neural Network Four Warning System and Its Application in Business Operation. Systems Engineering and Electronics, 1995,10: 50-58.

[8] Deng Julong. Grey Forecast and Grey Decision [M]. Huazhong University of Science and Technology Press, 2002.

[9] D. Luo, S.F. Liu. Dang Yaoguo. Gray Model GM (1,1) Optimization. China Engineering Science, 2003,08: 50-53.

[10] L.X. Zhang, J. Wang, etc. Human Resource Management Risk Early Warning Model based on BP Network. Nankai Business Review, 2007 (06): 78-85.

[11] Y.L. Zhang, Yang Naiding. Human Resource Risk. Fuzzy Comprehensive Evaluation Management Engineering, 2002 (01): 18-20. 